\title{
NARRATIVISME ET PHILOSOPHIE SPÉCULATIVE DE L'HISTOIRE
}

\author{
Maurice LagueuX ${ }^{1}$
}

Résumé: Au cours des dernières décennies, d'importants philosophes tels Walter B. Gallie, Arthur Danto, Louis Mink, Hayden White et Paul Ricœur ont mis l'accent sur le rôle de la narration en histoire. Le présent texte rappelle les thèses de ces auteurs et porte ensuite une attention particulière aux travaux de David Carr voulant que l'action historique elle-même ait une structure narrative. L'article discute des conséquences de ce «narrativisme » en prenant parti dans un débat alimenté par des interventions comme celles de William Dray et de Noël Carroll. Il soutient qu'une thèse " réaliste » en la matière risque de déboucher sur la réactualisation d'une forme de philosophie spéculative de l'histoire, ce qui ne va pas sans soulever quelques questions à propos de l'analyse politique et de la pratique de l'histoire.

MoTS-CLÉs : histoire, narration, récit, philosophie spéculative de l'histoire, réalisme, relativisme.

ABSTRACT: Over the last few decades, such important philosophers as Walter B. Gallie, Arthur Danto, Louis Mink, Hayden White and Paul Ricour have drawn attention to the role of narrative in history. After evoking the theses of these authors, this paper pays special attention to the works of David Carr which contend that historical action as such has a narrative structure. The paper discusses the consequences of such a "narrativism " and takes sides in the debate on this question which has been waged in part by William Dray and Noël Carroll. It is claimed that a "realistic" thesis on these matters might tend to generate the revival of a kind of speculative philosophy of history, which naturally would raise various questions concerning both political and historical analysis.

KEYWORDS : history, narrative, story, speculative philosophy of history, realism, relativism.

1. L'auteur tient à remercier de leurs utiles commentaires David Carr, Stéphan D'Amour, France Émond, Rajesh Mehta, Robert Nadeau et Lucas Sosoe. Il remercie également le Conseil de recherche en sciences humaines du Canada et le Fonds pour la formation de chercheur et l'aide à la recherche (Québec) de leur aide financière.

Revue de synthèse : $4^{e}$ S. $n^{0} 1$, janv.-mars 1998, p. 63-88. 
Zusammenfassung: In den letzten Jahrzenten haben wichtige Philosophen wie Walter B. Gallie, Arthur Danto, Louis Mink, Hayden White und Paul Ricour die Rolle der Narrativität in der Geschichte betont. Der vorliegende Text will zuerst die These dieser Autoren mit besonderer Berücksichtigung der Werke von David Carr darstellen. Dieser These entsprechend weist die geschichtliche Handlung eine narrative Struktur auf. Der Aufsatz diskutiert die Konsequenzen dieses "Narrativismus » und ergreift für Einwände wie diejenigen von William Dray und Noël Carroll Partei. Er vertritt den Standpunkt, daß eine " realistische " These in die Nähe einer Reaktualisierung einer Form von spekulativer Geschichtsschreibung rücken könnte, was in Bezug auf die politische Analyse und die praktische Geschichtsschreibung wichtige Fragen stellt.

STICHWÖRTER: Geschichte, Erzählung, Bericht, spekulativer Geschichtsphilosophie, Realismus, Relativismus.

Sommario: Nel corso degli ultimi decenni, filosofi importanti come Walter B. Gallie, Arthur Danto, Louis Mink, Hayden White e Paul Ricaur hanno posto l'accento sul ruolo della narrazione in storia. Nel presente testo vengono riportate le tesi degli autori, prestando particolare attenzione ai lavori di David Carr, per il quale è l'azione storica stessa ad avere una struttura narrativa. L'articolo discute le conseguenze di questo "narrativismo", schierandosi in un dibattito che è stato alimentato da interventi come quello di William Dray e Noël Carroll. Si sostiene che una tesi "realista sull'argomento sfocia nella riattualizzazione di una certa filosofia speculativa della storia, il che solleva alcuni problemi quanto all'analisi politica e alla pratica della ricerca storica.

Parole ChIAve: storia, narrazione, racconto, filosofia speculativa della storia, realismo, relativismo.

Maurice Lagueux, né en 1940, docteur en philosophie (Paris X-Nanterre), M.A. science économique (McGill), est professeur au département de philosophie de l'université de Montréal. Ses domaines de recherche sont la philosophie des sciences sociales (philosophie de l'économie, philosophie de l'histoire) et la philosophie de l'architecture.

Adresse : Département de philosophie, Université de Montréal, CP 6128, succ. Centre ville, Montréal, Québec H3C 3J7.

Courrier électronique : lagueuxm@ere.umontreal.ca 
L'importance qu'a prise, dans les études méthodologiques sur l'histoire au cours des années soixante-dix et quatre-vingts, la question de la structure narrative de l'ouvre historique peut paraître étonnante. Si l'on devait employer un langage inspiré par Imre Lakatos, on pourrait même dire que, au cours de cette période, le narrativisme a constitué une sorte de programme de recherche en nette progression, puisqu'il a donné lieu à une série de contributions qui ont, peu à peu et contre toute attente, conféré une étonnante respectabilité épistémologique à la narration, cette forme de discours si souvent discréditée depuis Descartes. Il ne saurait évidemment être question ici de rendre compte des multiples travaux rattachés au narrativisme, mais il peut être utile au départ de souligner l'importance de quatre contributions qui ont toutes eu pour conséquence d'accroître la crédibilité de la thèse narrativiste et de réduire la distance théorique qui sépare récits sans prétentions théoriques et activités généralement associées à la recherche scientifique. Bien que toute sélection ait quelque chose d'arbitraire, ces quatre contributions particulièrement décisives me paraissent être celles d'Arthur Danto, de Louis Mink, de Hayden White et de Paul Ricœur.

\section{L'ÉVOLUTION DES THÈSES NARRATIVISTES}

Si l'ouvrage que Danto a consacré à la philosophie analytique de l'histoire constitue une étape importante dans le développement du narrativisme, c'est que son auteur est parvenu à assurer une sorte de dignité épistémologique à l'idée toute simple voulant qu'une narration correcte constitue déjà une explication ${ }^{2}$. Si raconter correctement, c'est exposer de manière intelligible ce qui s'est passé lors d'une occasion donnée, n'est-ce pas par le fait même expliquer ce qui s'est passé en cette occasion? Sans doute, l'analyse des conditions de validité logique de l'explication ainsi véhiculée par une narration peut-elle nous renvoyer à la question de savoir ce qui confère un pouvoir explicatif à une telle explication, auquel cas sa

2. Pour plus de précisions sur les références citées en notes, se reporter à la bibliographie finale, p. 87-88. Ici, Danto, 1968, p. $201 \mathrm{sqq}$. Il parait juste de rapprocher de cette contribution celle de Walter B. Gall. dont l'ouvrage, GaLlie, 1964, essentiellement axé sur la question de la narrativité, est sensiblement contemporain de la première édition du livre de Danto. 
structure "narrative » pourrait alors se trouver réduite à une sorte de squelette formel. Toutefois, il ne sera pas question ici d'aborder cette question en tant que telle; il nous suffira de reconnaître que si la narration est à tout le moins porteuse d'explication, la distance qui sépare le texte narratif du texte théorique se trouve réduite d'autant.

Mink, pour sa part, s'est efforcé de présenter la narration comme un " instrument cognitif ${ }^{3}$ " après avoir, dès $1970^{4}$, distingué « trois modes de compréhension » qu'il désignait sous les noms de theoretical, categoreal et configurational et associait (en gros) respectivement aux sciences naturelles, à la philosophie et à l'histoire. Si le mode configurationnel se trouve ainsi associé à l'histoire, c'est qu'il se réalise dans le cadre de la narration, laquelle se voit ainsi élevée, à côté de la philosophie et de la science, au rang d'instrument rendant possible un nouveau type de compréhension et de connaissance.

Si les travaux de Danto et de Mink ont contribué à conférer une forme de respectabilité au récit historique comme mode d'explication ou de compréhension, ceux de White ${ }^{5}$ vont tendre à réduire la distinction traditionnelle entre les récits proposés par les historiens et ceux proposés par les auteurs littéraires et, de ce fait, à rendre quelque peu incertaine la frontière entre l'histoire et la fiction. Pour White, ce serait bien à tort que l'on réserverait aux seuls récits d'historiens les vertus cognitives qui sont désormais reconnues à la narration puisque, dans un cas comme dans l'autre, la structure narrative relève du narrateur lui-même et non de l'objet narré.

Toutes ces réflexions toutefois reposaient sur un postulat implicite selon lequel la forme narrative occupe une place décisive dans l'histoire des historiens. Mais les historiens contemporains, dans le sillage de l'école française des Annales, ont été de plus en plus nombreux à récuser la narration pour adopter plutôt une forme d'analyse de type interdisciplinaire où la place occupée par le récit traditionnel devient extrêmement marginale. Or la contribution de Ricœur ${ }^{6}$ a été précisément de montrer que, malgré les apparences, les travaux de ces historiens contemporains, qui sont loin de se présenter d'emblée comme des récits, n'en constituent pas moins des quasi-récits dans lesquels des quasi-événements concernent des quasipersonnages qui s'engagent dans des quasi-intrigues. Si la thèse narrativiste pouvait jusque-là être repoussée du revers de la main par ceux qui y voyaient une apologie d'une histoire événementielle généralement considérée comme dépassée, la contribution de Ricœur a été de rendre à tout le moins plausible l'idée selon laquelle la forme d'histoire la plus respectée,

3. MiNk, 1978 .

4. MINK, 1970 , p. 549-552.

5. Voir l'introduction de Whiтe, 1973 et WhITE, 1978.

6. RiceUR, 1983, p. 247-320. 
celle qui fut brillamment illustrée par les travaux des historiens de l'école des Annales, pouvait elle-même être structurée à la manière d'un récit, ou presque.

Au terme de ces développements, s'est donc imposée de plus en plus l'idée que toute œuvre historique valable offre une synthèse et une explication originales d'un aspect du réel, dans la mesure même où elle est structurée comme un récit, au même titre qu'une œuvre littéraire. Tout cela toutefois ne concernait que l'œuvre historique ou, plus précisément, l'œuvre historiographique, c'est-à-dire l'histoire en tant qu'elle est un texte écrit (ou, à la rigueur, un texte dit). Rien de ce qui est proposé par ces narrativistes ne concerne l'histoire réelle qui résulte des multiples actions humaines et qui constitue ce que l'on désigne souvent comme le passé de l'humanité. Les narrativistes s'intéressent, en effet, à l'histoire en tant qu'elle résulte de l'activité de l'historien, considérée comme activité « configurante » pour employer un terme de Mink, repris par Ricœur, qui traduit bien un aspect essentiel de la pensée de White. Bref, l'historien peut restructurer un monde réel qui, s'il faut en croire White, peut même, en tant que tel, être dit « informe ». Sans doute, Mink, Danto et Ricœur n'admettraient-ils pas aussi aisément que l'histoire réelle soit aussi amorphe, mais la liberté que ces auteurs reconnaissent à l'historien dans la mise en intrigue de son récit semble bien exiger que la réalité ne soit pas dotée ellemême des traits qui caractérisent une histoire, sans quoi l'historien n'aurait qu'à se faire le fidèle transcripteur de cette histoire qui se serait déroulée sans son concours. Mink expose cette idée en une phrase lapidaire : « En fait, il ne peut tout simplement pas y avoir d'histoires non racontées, de même qu'il ne peut y avoir de connaissance non connue ${ }^{7}$. " $C^{\prime}$ 'est, en un sens, ce qu'exprimait Danto au moment où il imaginait un « chroniqueur idéal » dont la connaissance des événements passés, toute complète et parfaite qu'elle soit par hypothèse, ne constituerait en rien un récit historique, faute de faire place à des intrigues qui, entre autres choses, relieraient ces événements connus à des événements postérieurs encore inconnus ${ }^{8}$. Quant à Ricour, il n'hésite pas à privilégier l'emploi de termes actifs comme « mise en intrigue » et « agencement » plutôt que des termes plus passifs comme « intrigue » et «système » pour mettre en relief que c'est bien à une véritable « opération de configuration ${ }^{9}$ » qu'on a affaire. Bref - et c'est ce que David Carr, qui, comme on le verra, défend une thèse tout opposée, reproche à ces auteurs ${ }^{10}$-, la narration historique n'irait pas, à leurs yeux, sans que le narrateur prenne sur soi de conférer - d'« impo-

7. Mink, 1978, p. 201.

8. DANTO, 1968 , p. 149 sqq.

9. RiCEUR, 1983, p. 102.

10. CARR, 1985, p. 302 sqq. et l'introduction de CarR, 1986. 
ser », préfère dire Carr - une « forme " à une réalité historique qui, en tant que telle, serait passablement amorphe.

Sans doute, une telle approche n'interdit pas vraiment de distinguer histoire et fiction comme Danto, Mink et Ricœur l'admettraient volontiers et comme White le concéderait sans doute, quoiqu'un peu moins résolument. Pour maintenir un écart décisif entre histoire et fiction, il suffit, en effet, de reconnaître que l'historien ne doit évoquer que des faits bien établis. Cela, toutefois, n'empêche pas de soutenir que l'intrigue, qui donne un sens unifié à une telle succession de faits, est "imposée " en quelque sorte à ces faits par l'historien, bien plus qu'elle n'en est dégagée. Il est vrai que Ricœur insiste sur le fait que l'intrigue n'est pas imposée à la réalité historique de manière purement artificielle puisque, selon lui, elle est en quelque sorte "préfigurée " dans le monde de l'action, mais il se garde bien d'en conclure que ce monde de l'action humaine est lui-même doté d'une structure proprement narrative ${ }^{11}$. Pour éviter de prêter carrément une structure narrative aux actions humaines et à la réalité historique tout en soutenant qu'il serait néanmoins possible d'entrevoir dans cette réalité historique la " préfiguration » d'une intrigue, Ricœur fait valoir que l'ensemble de faits susceptible de faire l'objet d'une configuration narrative doit posséder certains traits qui seront alors appelés «pré-narratifs ». En somme, l'historien ne saurait faire l'histoire de n'importe quoi. Pour s'en convaincre, on n'a qu'à penser au fait que la nature, comme le disait Hegel, n'a pas d'histoire ${ }^{12}$. Certes, la nature est-elle structurée d'une certaine façon et on peut en faire l'« histoire » au sens où l'on peut décrire son évolution, mais elle ne peut constituer l'objet d'une histoire au sens où les faits et gestes des êtres humains le peuvent. Même si tel n'est pas le contexte de la démarche de Ricœur, c'est, me semble-t-il, quelque chose de ce genre qu'il suggère quand, dans son analyse de ce qu'il appelle Mimesis $\mathrm{I}$, il se réfère à la «structure pré-narrative» du monde pourtant encore inchoatif de l'action ${ }^{13}$. Ce monde ne serait donc pas une masse informe, comme le suggère parfois White, et il ne serait pas davantage une pure succession d'instants atomiques - ce qui, en un sens, reviendrait au même car, dans les deux cas, il y aurait alors négation d'une véritable structure temporelle ou, si l'on préfère, d'une véritable historicité.

Toutefois, il est difficile de voir en quel sens on pourrait qualifier de "pré-narratif » le monde de l'action humaine, si ce n'est pour souligner qu'il est inséparable d'une structure temporelle qui, comme l'a éloquemment rappelé $\mathrm{Carr}^{14}$, peut seule rendre l'histoire possible. Est-ce à

\footnotetext{
11. Sur ce point, voir Riceur, 1983, p. 85-87 et RicGur, 1985, p. 316-318.

12. Hegel, 1939, t. I, p. 247.

13. Voir, par ex., RicEUUR, 1983, p. 113.

14. Voir, par ex., Carr, 1991, l'introduction de CARR, 1986, ainsi que Carr, 1985, p. $303-304$.
} 
dire que l'on peut faire le pas de plus auquel Ricour semblait résister et affirmer que ce monde de l'action, qui est le lieu même où l'histoire se réalise, est doté lui-même non seulement d'une structure temporelle mais d'une structure proprement narrative? Bref, si l'on refuse à l'historien le droit et la capacité d' « imposer »- pour reprendre à nouveau le terme adopté par Carr ${ }^{15}$ - une structure narrative à un quelconque secteur du monde réel qui, en tant que tel, y serait étranger, faut-il en conclure que les intrigues exposées par l'historien dans son récit existent préalablement dans le monde réel de l'action humaine? À cette question, Carr n'hésite pas à répondre par l'affirmative, en soutenant, au nom d'une sorte d'exigence de clarté conceptuelle, que la réalité dont on fait l'histoire est elle-même structurée à la manière d'un récit ${ }^{16}$. Avant de voir si une telle thèse est défendable et de chercher à préciser quelle forme elle pourrait prendre, demandons-nous ce qu'il faut entendre par un récit ou une narration.

\section{QU'EST-CE QU'UN RÉCIT?}

Plus précisément, demandons-nous quels sont les traits structurels qu'il faudrait retrouver dans la réalité historique pour que l'on puisse conclure qu'elle est elle-même structurốe à la manière d'un récit. Il est assez habituel, du moins chez les auteurs dits « narrativistes » dont il est ici question, de dégager de la Poétique d'Aristote les traits essentiels qui caractérisent le récit. Le récit est alors identifié à ce qu'Aristote appelle muthos, terme que l'on traduit volontiers par « histoire», au sens anglais de story. Aristote y définit d'abord l'histoire comme «l'agencement des actes accomplis ${ }^{17}$ »; toutefois, cette première définition se trouve vite précisée par l'idée que si l'histoire concerne des actes ou des actions, ces actions doivent être des actions « formant un tout ${ }^{18}$ ». Aristote s'explique d'ailleurs en ces termes : « $[\ldots]$ et les parties constituées des actes accomplis doivent être agencées de façon que, si l'on déplace ou supprime l'une d'elles, le tout

15. CARr, 1985, p. 302 et CARr, 1986, p. 15.

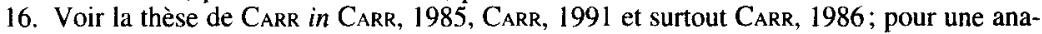
lyse attentive de ce dernier ouvrage, voir DraY, 1989, chap. vII, part. II. Dans CARr, 1986, p. 16-17, David Carr cite quelques auteurs qui soutiendraient comme lui qu'il y a une sorte de continuité entre le monde de la narration et celui de la vie quotidienne et de l'action. Carr semble toutefois disposé à admettre qu'il vaudrait peut-être mieux se contenter de parler de traits quasi-narratifs quand il est question du monde de l'action.

17. ARISTOTE, 1990, 1450 a 4-5.

18. Aristote, 1990,1451 a 33. 
soit troublé et bouleversé ${ }^{19}$. " Or, comme Aristote l'avait précisé plus tôt, " forme un tout, ce qui a commencement, milieu et fin ${ }^{20}$ ". Et c'est là le trait qui est généralement retenu pour caractériser le récit : celui-ci forme un tout et, en ce sens, a un commencement, un milieu et une fin. L'objet du récit peut être la façon dont des actes accomplis dans la réalité ont pu être effectivement agencé, mais l'important est que cet agencement puisse être compris comme une totalité dont il y a un certain sens à dire qu'elle est « achevée ».

Un deuxième trait caractéristique du récit est encore plus fondamental puisqu'il s'agit de ce qui constitue, en quelque sorte, le cœur même de l'histoire (story) qui fait l'objet du récit. Puisque le mot «histoire» en français ne permet pas de distinguer l'histoire entendue au sens de story de l'histoire entendue au sens d'historiographie, Ricœur choisit de traduire par « intrigue » (« sur le modèle du terme anglais plot ${ }^{21}$ ») cela même qu'Aristote désignait par le terme muthos. Ce qui est suggéré ainsi, c'est qu'il y a narration là où est racontée une histoire qui tient son unité du fait qu'elle est animée par une intrigue. Ce second trait est d'ailleurs' difficilement dissociable du premier. Comment, en effet, un ensemble d'actions pourrait-il former un tout avec commencement, milieu et fin si ce n'est par le fait que ces actions sont reliées par l'intrigue dont elles sont des éléments, en ce sens que, considérées ensemble, elles permettent de comprendre que quelque chose qui n'allait pas de soi s'est réalisé — « selon la vraisemblance ou la nécessité », comme dirait Aristote ${ }^{22}$. C'est en ce sens, en tout cas, que l'on dira qu'il y a narration quand des faits et gestes se trouvent reliés par le déroulement d'une intrigue. Il est possible que cette intrigue se réduise à assez peu de choses. Elle pourrait bien, en effet, ne consister qu'en un processus expliquant qu'un changement quelconque soit advenu quelque part, mais l'important est que cette intrigue aboutisse clairement à un dénouement comme celui que constitue l'avènement de ce quelque chose.

Enfin, un troisième trait propre au récit a été utilement mis en lumière par Danto dans son analyse de la structure narrative des textes historiques. Il s'agit de la présence de ce que Danto appelle les «propositions narrative ${ }^{23}$ » et qu'il définit comme des propositions à l'aide desquelles un événement E-1 peut être décrit à l'aide d'un autre événement E-2 qui a eu lieu ultérieurement. L'exemple qui est devenu le cas paradigmatique de ce type de proposition est le suivant : "en 1713 naissait l'auteur du Neveu de Rameau », où la naissance d'un enfant en 1713 est décrite, par la personne

19. ARISTOTE, 1990,1451 a $33-35$.

20. Aristote, 1990,1450 b 26-27.

21. RiCEUR, 1983 , p. 57 , n. 2.

22. Aristote, 1990,1451 a 15,1452 a 24 ; cf. aussi : 1451 a 39,1454 a 34.

23. Danto, 1968, chap. VIH; pour la définition, voir p. 152. 
qui narre cet événement, à la lumière d'un autre événement survenu beaucoup plus tard, soit la rédaction d'un ouvrage par l'écrivain que cet enfant était alors devenu. Ce trait est aussi étroitement relié aux premiers puisqu'il dérive du fait que le narrateur raconte une histoire dont il connaît déjà le commencement, le milieu et même la fin de telle sorte que, pour rendre compte de l'intrigue qui constitue le cœur de cette histoire, il est constamment amené à souligner que les événements qui ont lieu à un moment donné de cette histoire prennent leur sens, du point de vue de cette intrigue, à la lumière d'autres événements qui ont lieu plus tard dans son développement.

LE MONDE DES ACTIONS HUMAINES EST-IL STRUCTURÉ À LA MANIÈRE D'UN RÉCIT?

Si tels sont les traits du récit, peut-on encore parler avec Carr d'une structure narrative qui serait déjà présente dans la réalité avant même que celle-ci soit racontée par un historien? Certes, l'idée aristotélicienne voulant que l'histoire ou l'intrigue concerne des « actes accomplis » pourrait laisser penser qu'il faut répondre à cette question par l'affirmative puisque ces actes sont bel et bien accomplis dans le monde réel. Toutefois, le fait qu'Aristote mette l'accent sur «l'agencement» de ces actes plus que sur les actes eux-mêmes rend déjà les choses plus complexes. Sans doute, aurait-on bien tort de comprendre l'action humaine comme une réalité ponctuelle et atomique et c'est l'un des mérites des travaux de Carr que d'avoir souligné avec force le fait que les actions humaines en tant que telles soient justement « agencées ». S'appuyant sur l'analyse phénoménologique de la temporalité qu'a développée Husserl alors qu'il montrait que le présent est inséparable d'une protention vers l'avenir et d'une rétention du passé, puis retrouvant, dans la même lancée, l'intuition fondamentale de Heidegger à propos de l'historicité de l'être humain, Carr montre de façon convaincante qu'une analyse de l'action humaine est indissociable d'une analyse de la temporalitée ${ }^{24}$. Et si une action menée à sa modeste échelle s'inscrit ainsi dans une structure temporelle, cette action s'intègre à un projet plus vaste de telle sorte que, à l'échelle plus large qui intéresse l'histoire, les actions humaines se trouvent agencées au sein même de cette structure temporelle qui donne sens à une vie humaine ou, si l'on préfère, à une biographie au sens le plus fort du terme ${ }^{25}$. Reste pourtant à savoir si

24. Carr, 1991, p. 208-211, Carr, 1986, p. 3-7, ainsi que Carr, 1985, p. 303.

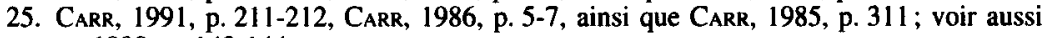
Carrol l, 1990 , p. 143-144. 
cette structuration temporelle des actions humaines suffit à leur conférer un caractère proprement narratif.

D'abord, peut-on dire que l'agencement d'actions humaines qui vient d'être évoqué constitue un tout qui a un commencement, un milieu et une fin? Bien sûr, toute action - ou tout agencement d'actions - commence quelque part et finit quelque part. Dans le flux du temps, tout point peut arbitrairement être considéré comme le commencement de ce qui va suivre ou comme la fin de ce qui a précédé. Mais voir, de ce fait, autant de narrations dans chaque segment de temps serait évidemment vider de son contenu la thèse aristotélicienne sur le commencement, le milieu et la fin puisqu'il y est question du commencement et de la fin de quelque chose qui constitue un tout. Or, en ce sens, la fin doit constituer une clôture et donc n'être pas suivie d'autre chose, ou mieux, ne pas appeler une suite, au sens où le «milieu » de l'histoire en appelle une. Toute action humaine, il est vrai, est orientée vers une fin ${ }^{26}$. Mais il s'agit d'une fin au sens de « finalité » et non de «clôture ». La fin-finalité d'une action n'est pas une finclôture car la finalité s'intègre habituellement à une finalité plus large; elle n'a rien de la fin d'une histoire. Certes, la réalisation d'une fin peut-elle constituer une clôture, mais si toute action humaine est orientée vers une fin, toute action humaine ne consacre pas la réalisation d'une fin.

Rien pourtant ne m'empêche d'appréhender un ensemble d'actions ou même une action isolée, fût-ce la plus modeste, comme une totalité signifiante. Il suffit pour ce faire de reconnaître dans cette action (ou, le cas échéant, dans cet ensemble d'actions agencées) le développement d'une intrigue. Je prendrai pour exemple une action ultrabanale car il n'y a pas de critères qui permettraient de distinguer parmi les actions (ou ensemble d'actions agencées) celles dont on peut faire l'histoire de celles qui seraient trop insignifiantes pour qu'on en fasse l'histoire. Considérons donc l'exemple suivant : je prends mon crayon (qui était sur la table). Cette action est, en quelque sorte, perdue dans une forêt d'actions (simultanées ou pas) d'importance à tout le moins comparable. Pourquoi faudrait-il voir dans l'état du monde où le crayon est sur la table le début d'une histoire et dans celui où il est dans ma main la fin de cette histoire? Cette présumée fin est tout aussi bien le début d'une action qui suit la prise en main du crayon et ainsi de suite. Aussi n'est-ce la fin de quelque chose que dans la mesure où une intrigue se dénouerait avec la prise en main du crayon. De fait, rien n'empêche de voir là la conclusion d'une mini-intrigue. Après tout, j'ai pu avoir quelque mal à prendre ce crayon qui a furtivement roulé sous mes doigts; j’aurais pu le laisser échapper, mais voilà, en glissant

26. Il semble d'ailleurs que Carr estime pouvoir tirer parti de ce caractère finalisé des actions humaines; voir, par ex., CARR, 1985, p. 304. 
prestement mon index tout en faisant pivoter mon poignet, j'ai pu le retenir fermement de telle sorte que s'est ainsi trouvé bien instauré l'état du monde dans lequel je tiens le crayon, ce qui clôt avec bonheur cette histoire dont il ne faudrait certes pas exagérer l'importance ou le côté palpitant, mais qui n'en est pas moins une histoire pour autant.

Faut-il alors conclure du fait que la moindre action peut donner lieu à une intrigue que le monde des actions est doté d'une structure narrative ? Il n'y a aucune raison de le penser car ce qui vient ici d'être présenté comme une action se déroulant dans le monde réel n'est rien d'autre qu'une histoire racontée par moi-même en tant qu'observateur de ma modeste action ou, si l'on préfère, par moi-même qui me fait prétentieusement historien d'une infime parcelle de ma vie. En l'occurrence, le narrateur aurait d'ailleurs tout aussi bien pu être un autre, voire un historien professionnel qui, contre toute attente, se serait intéressé à une action aussi insignifiante. De ce point de vue, ma narration se compare, toute proportion gardée, à celle d'un historien qui écrit l'histoire d'une guerre. Tout comme il n'y a rien d'absurde à soutenir que cette histoire de la guerre en question résulte du fait qu'un historien a su mettre en intrigue un ensemble d'événements qui ont eu lieu avant, pendant et après les affrontements qui ont constitué cette guerre, il n'y a rien d'absurde à dire que l'histoire de la prise en main du crayon résulte du fait que j'ai procédé à son propos, au moment où j'ai estimé indiqué d'en faire l'histoire, à une mise en intrigue d'un ensemble de mini-événements associés à cette action. Sans m'attarder à la question de savoir si cette dernière mise en intrigue s'est effectuée sur un mode « comique » plutôt que sur un autre des modes distingués par White, je conclurai de cette réflexion que le fait qu'une action puisse être décomposée en un ensemble de mini-actions (plus ou moins spontanées) susceptibles d'être pensées dans le cadre d'une mini-intrigue n'implique en rien que l'action elle-même soit dotée d'une structure narrative.

Il est vrai, comme Carr l'a bien fait observer, que la réalisation, à travers une suite d'actions, d'un plan conçu par un agent historique peut constituer l'essentiel d'une authentique intrigue, à condition toutefois que la réalisation de ce plan soit pensée à la manière d'une intrigue. Elle peut l'être, soit par l'agent lui-même au moment où il met en œuvre son plan, soit par un historien qui choisit de voir dans cet ensemble d'activités les grandes lignes d'une intrigue. Occasionnellement d'ailleurs - qu'on pense à Jules César qui devait rédiger sa Guerre des Gaules et au général de Gaulle qui devait rédiger ses Mémoires - l'agent historique qui pense son plan d'action et l'historien qui présente ensuite ce même plan comme le déroulement d'une intrigue ne font qu'une seule et même personne. Rien, toutefois, n'autorise à penser que la réalisation d'un tel plan à partir d'un ensemble d'actions porte en soi une intrigue qui devrait s'imposer à l'historien. Comment 
pourrait-on expliquer autrement que ces actions peuvent être tout aussi bien intégrées à une foule d'intrigues indépendantes les unes des autres et indépendantes de celle que l'agent historique avait en vue au moment où il concevait son plan? Mais examinons la chose de plus près.

Dans un échange qui a eu lieu à l'université d'Ottawa à propos de l'ouvrage de Ricœur, Temps et récit, ce dernier faisait observer à Carr que " quand nous tentons aujourd'hui de comprendre Lénine, nous ne pouvons pas le faire uniquement sur la base de ce qu'il a conçu comme devant être la révolution bolchevique, mais aussi à la lumière du fait qu'il a produit Staline $^{27}$ ». Ce que Ricœur voulait souligner par là, ce n'est pas que les " effets pervers » qui résultent d'une action importeraient plus par principe que les effets intentionnels, c'est, plus modestement, qu'ils importent, à tout le moins, tout autant. On ne peut rien objecter à Carr quand il soutient qu'une vie bien vécue - que ce soit celle de Lénine ou celle du général de Gaulle - peut se dérouler et se dénouer à la manière d'une passionnante intrigue; mais on doit admettre que les actions que posent ceux qui vivent cette vie peuvent tout aussi bien, et à un titre équivalent, constituer les éléments d'une multitude d'autres intrigues. On a rappelé que maints héros de l'histoire ont eu tendance à se faire eux-mêmes, par anticipation, les historiens de leur propre vie, en conduisant celle-ci selon un certain plan de vie qu'ils dégagent ensuite sous forme d'intrigue au moment où ils écrivent leurs mémoires; mais il suffit d'admettre que la même vie peut être très légitimement pensée autrement pour que l'intrigue qui inspirait le héros historien de lui-même perde tout privilège du point de vue de l'histoire qu'il convient d'en faire. Il ne saurait en être autrement si l'on admet que les acteurs de l'histoire ne peuvent contrôler les événements extérieurs qui viennent modifier d'une manière décisive leurs plans de vie ni les interactions humaines qui viennent donner autant de sens différents à leurs actions. Plus exactement, la façon dont le héros a pensé sa propre vie devient, dans cette perspective, un élément parmi d'autres dans la masse de ses faits et gestes, que les historiens intégreront légitimement à de nouvelles intrigues.

Par exemple, quand le général de Gaulle a lancé en 1967 son célèbre «Vive le Québec libre » du haut du balcon de l'hôtel de ville de Montréal, il était apparemment inspiré par un plan quelconque dont on pouvait s'attendre à trouver l'écho dans ses Mémoires. Du coup, un biographe du Général pouvait choisir de raconter son histoire en se laissant guider par une intrigue dont le déroulement coïnciderait exactement avec celui du plan de De Gaulle (que l'on supposera ici clairement consigné dans les Mémoires), voire avec l'ensemble de son plan de vie (dont on concédera

27. RiceUR, 1985, p. 317. 
même que, pour l'essentiel, il aurait été exceptionnellement bien réalisé). Mais ce n'est là que l'une des intrigues possibles parmi celles qui peuvent soutenir une histoire de De Gaulle et il n'y a pas de raison de penser que ce soit la plus valable. C'est là l'essentiel de l'objection de Ricour illustrée par l'exemple de Lénine et de son rapport au stalinisme. Les effets pervers et les « effets non voulus » - qui, par définition, échappent au contrôle des agents historiques - peuvent également jouer un rôle décisif au sein des intrigues dont ces agents historiques sont les acteurs privilégiés. D'ailleurs, il est bien connu que, à mesure que se développent tant les événements que les connaissances historiques, ces intrigues sont soumises à de constantes révisions de la part des historiens. Selon que l'indépendance du Québec se réalisera ou pas dans un avenir encore indéterminé trente ans après cet événement, le cri du Général sera présenté dans une centaine d'années ou bien comme un geste prophétique qui a donné un solide coup de pouce à l'histoire et secoué des esprits hésitants qui devaient s'engager progressivement sur une voie irréversible, ou bien comme un simple coup d'épée dans l'eau dont les séquelles devaient tout au plus contribuer pendant quelques décennies à brouiller les cartes et à retarder l'inévitable intégration du Québec à l'ensemble canadien et nord-américain.

Qui plus est, les actions des agents historiques peuvent aussi s'intégrer non moins légitimement à une foule d'autres intrigues qui ont très peu à voir avec la vie de leurs auteurs. Par exemple, le geste de De Gaulle à Montréal peut constituer un élément susceptible en principe de s'intégrer à une infinité d'intrigues différentes qui ne constituent pas forcément des « vies » ou des « histoires » de De Gaulle. On peut voir dans le cri du Général un moment plus ou moins déterminant dans l'histoire de ses hôtes canadiens ou dans celles d'un nationaliste québécois enthousiasmé par cet appui inattendu, d'un automobiliste gêné par les rassemblements qui ont suivi ce moment historique, du balayeur obligé de nettoyer les abords de l'hôtel de ville au lendemain de l'événement, etc. Dans chaque cas, on a affaire à des intrigues différentes dont il serait abusif de dire qu'elles préexistent dans la réalité du seul fait que leurs éléments sont tirés du monde des actions réelles. Admettre que les actions réelles sont susceptibles d'être intégrées à la trame d'un nombre essentiellement indéfini d'intrigues, c'est admettre que ces intrigues ne sauraient constituer, en tant que telles, des éléments constitutifs de la structure de ce monde réel. Pour penser autrement, il faudrait admettre que cette structure qui est censée exister réellement dans le monde réel n'est qu'une structure potentielle pouvant légitimer une infinité de récits potentiels qui, s'ils étaient tous actualisés, renverraient à l'enchevêtrement d'une multitude de relations causales s'entrecroisant à l'infini pour former le chiasme inénarrable des influences et des contre-influences qui se sont exercées à chacun des moments de l'histoire. 
Sans doute faut-il reconnaître avec Noël Carroll que les diverses histoires qui sont ainsi arrachées à ce chiasme par les historiens qui choisissent d'en faire le récit peuvent être compatibles entre elles et qu'en ce sens elles pourraient, en principe, être toutes littéralement vraies ${ }^{28}$. Toutefois, ce qui est en cause ici n'est pas, à proprement parler, la compatibilité ou même la vérité des histoires proposées par les historiens. On l'a vu, on peut parler d'une histoire vraie, par opposition à une fiction, si tous les énoncés factuels dont cette histoire se compose sont vrais. Mais, on l'a vu également, un récit n'est pas constitué uniquement d'énoncés, mais bien d'une structure dont l'élément essentiel est une intrigue qui nous conduit d'un commencement à une fin. Aussi, ce qui est en cause ici est la question de savoir si une telle structure se retrouve ou pas dans la réalité. Certes, si on a affaire à des récits historiques sérieux, les éléments constitutifs de l'intrigue refléteront-ils des enchaînements entre un ensemble de causes réelles et les effets que l'historien aura su leur imputer. Ce n'est pas parce que le mot du général de Gaulle a pu avoir une foule d'autres effets que ceux que l'historien choisit de lui imputer ne sont pas parfaitement réels. De même, pour reprendre un exemple de Carroll, Pearl Harbor a bel et bien causé réellement l'entrée en guerre des États-Unis, quelles que soient les multiples autres conséquences de cet important événement. Pourtant, puisque le monde réel n'est qu'un tissu inextricable d'enchaînements de causạlité indénombrables, affirmer que les enchaînements mis en relief par l'historien étaient déjà présents dans le monde réel équivaut à peu près à affirmer, comme Michel-Ange l'aurait fait, que ses statues étaient déjà présentes dans les blocs de marbre informes auxquels il suffisait après tout d'enlever les morceaux qu'ils contenaient en trop! Un peu comme MichelAnge arrachait ses statues à la masse informe de la pierre tout en respectant les veines qu'il choisissait de mettre en valeur, l'historien arrache son récit au chiasme informe des relations causales tout en respectant, bien sûr, ceux de ces enchevêtrements qu'il choisit de mettre en relief. L'historien dégage donc quelque chose que l'on peut considérer comme une structure, mais qu'a-t-on gagné à soutenir qu'il s'agit d'une structure narrative si l'on doit reconnaître aussitôt que cette présumée structure n'est, somme toute, qu'une structure virtuelle dont les divers éléments ne sauraient s'actualiser sans que des historiens choisissent d'en faire le récit?

À la limite, on pourrait même craindre de voir cette structure toute virtuelle se diluer en une réalité totalement amorphe et susceptible de donner lieu aux interprétations les plus fantaisistes, mais ce serait là une crainte excessive. Rien, en effet, de ce qui vient d'être dit n'implique que l'historien peut à loisir inventer les intrigues qui lui plaisent et les « imposer » à

28. Carroll, 1990, p. 153-154; voir aussi ibid., p. 145-146. 
la réalité. Les intrigues que peut invoquer l'historien ne sont pas arbitraires puisqu'elles doivent prendre appui sur des faits qui doivent être bien établis et sur des relations entre ces faits qui doivent être bien fondées. Peut-être est-ce en ce sens que William Dray soutient que, au lieu de dénoncer l'antiréalisme de White en cherchant, comme Carr semble vouloir le faire, à réhabiliter contre Mink l'idée selon laquelle des « histoires non racontées » existeraient bel et bien dans le monde des actions réelles, il vaut mieux se contenter plus modestement de soutenir qu'on y trouve à tout le moins des « configurations narrativisables encore inconnues ${ }^{29}$ ». On peut soutenir, en effet, que le réel n'est pas une masse amorphe sans admettre pour autant que ce qui s'y passe est déjà structuré au sens où les récits le sont. Il suffit pour cela de faire valoir que tout ne peut être dit à son propos, que tout n'est pas «narrativisable», que seules certaines configurations (ou réseaux de relations entre événements réels) le sont, même si l'on peut soutenir par ailleurs que ces configurations sont littéralement indénombrables. Sans doute est-ce au nom d'une telle façon de voir, nettement moins radicale que celle de Carr, que Dray reprochait à Mink de comparer non sans ironie la notion d' " histoires non racontées » à celle manifestement impensable de " connaissance inconnue » et proposait de la rapprocher plutôt de celle, parfaitement acceptable, de « faits non établis $"{ }^{30}$. Dray faisait ressortir ainsi le caractère quelque peu abusif de la comparaison de Mink dans la mesure où, par définition, une connaissance ne peut exister sans être connue alors qu'on ne peut affirmer a priori qu'une histoire ne peut exister sans être racontée. Toutefois, il se rendait la partie un peu trop facile dans la mesure où les faits (bien établis ou pas) constituent, par définition, les ingrédients du monde réel alors qu'on ne peut affirmer a priori que les histoires en constituent des ingrédients au même titre. Toute la question celle qu'en fait pose Carr - est donc de savoir si les configurations de fait qui sont susceptibles de donner lieu à une narration peuvent être considérées ou pas comme une partie constitutive du monde réel. Or la réponse de Dray est qu'elles pourraient l'être, mais dans la mesure seulement où l'on parle de configurations qui ne seraient pas encore « narrativisées ». Une telle position suffit à contrer l'antiréalisme puisqu'il y est maintenu que les histoires ne peuvent trouver leur source que dans le monde réel, mais elle ne suffit pas à donner raison à un strict réalisme comme celui dont se réclame Carr puisqu'une réalité aussi floue qu'une configuration de faits qui n'aurait pas encore fait l'objet d'une narration pourrait fort bien n'être rien d'autre qu'un ensemble de faits et de relations qu'un historien éventuel pourrait librement découper dans le chiasme indéfini de relations que j'ai décrit plus haut.

29. DraY, 1989, chap. VIl, « Narrative and Historical Realism », p. 162.

30. Dray, 1989, chap. vil, p. 161-162. 
Si l'on adopte cette forme très modérée de réalisme qui permet de soutenir que les diverses intrigues possibles qui « appartiennent » au monde réel demeurent rigoureusement indénombrables dans la mesure où elles n'appartiennent à ce monde réel qu'en tant qu'ensembles de faits et de relations pouvant potentiellement être extraits du chiasme (décrit ci-dessus) par un historien qui en dessinerait littéralement la configuration, on peut reprendre à nouveaux frais le constat paradoxal de Mink à propos de l'impossible agrégation des intrigues. Mink fait observer que si l'on comprend aisément que deux récits de fiction concernant un même personnage ne puissent être agrégés et former un tout, on comprend mal qu'il en aille de même de récits historiques ayant trait à des personnages réels, et pourtant les récits des historiens ne peuvent typiquement pas être agrégés les uns aux autres ${ }^{31}$. Dray lui répond que cette exigence d'agrégation est bien excessive et qu'elle se ramène à une exigence d'objectivité que l'histoire, bien sûr, ne peut satisfaire; mais il ajoute aussitôt que ce problème, somme toute assez banal, n'a rien à voir avec son caractère narratif ou pas $^{32}$. Pourtant, si ce problème n'en est pas vraiment un quand on adopte une position réaliste très modérée comme celle que je décris ici, il me semble être décisif si l'on adopte une position réaliste radicale comme celle de Carr. Rien ne s'oppose, en effet, à ce qu'une foule d'intrigues concernant les mêmes personnages coexistent dans l'esprit d'historiens qui s'emploient à dégager des configurations variées et fort partielles, voire un chiasme indescriptible composé d'une multitude de relations déployées à une multitude de niveaux. Ces intrigues n'existeraient pas en tant que telles dans la réalité mais elles seraient toutes formées à partir de découpages variés dans un ensemble infiniment complexe. Dans un tel cas, il n'y aurait manifestement aucun sens à exiger que de telles intrigues soient susceptibles de s'intégrer dans une totalité unique. Tout est différent cependant si l'on soutient que ces intrigues coexistent comme telles dans le monde réel, car il n'y aurait plus de sens alors à parler d'intrigues autonomes, indénombrables et constamment révisables à la lumière d'événements futurs. À quoi servirait-il, en effet, d'assurer que ces intrigues constituent la structure même de l'histoire réelle si c'était pour reconnaître aussitôt qu'elles n'occupent pas une place bien définie dans la structure d'ensemble de cette histoire réelle, qu'il n'est pas possible de les circonscrire vraiment et qu'elles pourraient, avec le temps, se révéler être autres que ce qu'elles sont au moment présent? Si ces intrigues constituent ensemble la structure même de l'histoire réelle, c'est que l'histoire réelle $a$

31. Mink, 1978, p. 195-197.

32. Dray, 1989, chap. vil, p. 158-159. 
une structure ou, si l'on préfère, qu'il existe quelque chose comme une histoire réelle ou quelque chose comme une Histoire universelle ${ }^{33}$.

RÉALISME HISTORIQUE ET PHILOSOPHIE SPÉCULATIVE DE L'HISTOIRE

On pourrait, en effet, dire sans équivoque que l'histoire réelle est dotée d'une structure narrative qui lui appartient vraiment si elle pouvait, en tant que telle, être l'objet d'un récit unique, totalisant et définitif. Cette histoire se donnerait alors comme rien moins que le Récit d'un Narrateur universel qui en contrôlerait toutes les variables. L'ensemble des actions humaines s'intégreraient alors, tout au long de l'histoire, à une intrigue fondamentale qui s'identifierait au Plan de ce Narrateur. Or, une telle intrigue est très précisément ce que ceux qui ont développé une théologie chrétienne de l'histoire entendaient retrouver grâce à une lecture attentive de l'histoire humaine. Pour ces théologiens de l'histoire, l'histoire réelle était rigoureusement conçue comme un récit. Cette histoire avait un commencement (raconté par la Genèse), un milieu (dont la Rédemption de l'humanité par la mort du Christ constituait le moment essentiel) et une fin (annoncée par des textes apocalyptiques). Pour eux, une fois intégrées à cette intrigue fondamentale, toutes les actions des acteurs de l'histoire trouvaient leur sens véritable, même si ce sens était bien rarement entrevu par ces acteurs qui ne pouvaient normalement en reconnaître les moments essentiels puisque les secrets de Dieu demeurent insondables. Quand César traverse le Rubicon, il n'est guidé que par son ambition et son intérêt, mais, expliquerait alors Bossuet, il n'est, en réalité, qu'un instrument entre les mains de Dieu qui veut, à travers lui, amorcer le processus qui débouchera sur la création de l'Empire romain, lequel ne sera lui-même que l'instrument destiné à rendre possible l'affirmation quasi universelle de la chrétienté. Aussi, ceux qui ont exposé cette théologie de l'histoire n'ont-ils pas manqué de recourir couramment à des «propositions narratives » au sens de Danto. C'est ainsi, par exemple, que cette théologie de l'histoire nous raconte avec force détails comment fut sauvé des eaux l'enfant qui, plus tard, devait sortir son peuple d'Égypte et, bien sûr, nous fait assister à l'humble naissance de Celui qui devait mourir sur la croix pour sauver le monde.

33. C'est bien ce que suggère Louis Mink à la suite de sa discussion de l'agrégation, voir Mink, 1978, p. 196, mais c'est aussi ce que conteste Noël Carroll en mettant l'accent, comme on l'a vu, sur la question de la compatibilité des histoires et de leur vérité et en alléguant, de ce point de vue, qu'une histoire peut évidemment être dite vraie sans qu'il soit question pour autant d'une Histoire à prétention universelle; voir Carroll, 1990, p. 152-154. 
Bref, les théologiens de l'histoire ont constamment cherché à lire une intrigue déjà pleinement présente dans l'histoire réelle, et cela était, de leur part, parfaitement cohérent puisque l'histoire réelle était conçue par eux comme un grand livre, le Livre de la Création, que Dieu lui-même avait écrit et qu'il s'agissait de décrypter à l'aide d'un autre livre, la Bible, qui était présumé en révéler les lignes essentielles. Pour eux, il s'agissait éminemment de découvrir les intrigues qui structuraient l'histoire réelle et non d'imposer à celle-ci des intrigues qui lui auraient été étrangères. C'est ainsi, par exemple, que saint Augustin, fasciné à la fois par la durée et par la chute éminente de l'Empire romain, propose à ses lecteurs d'examiner «pourquoi le vrai Dieu qui tient dans sa main tous les États de la terre daigna favoriser l'accroissement de l'Empire romain ${ }^{34}$ ». De même Bossuet, chargé d'initier un futur roi de France aux secrets de l'histoire universelle, propose de chercher dans les Saintes Écritures la clé qui peut seule permettre de les percer: «Les jugements de Dieu sur le plus grand de tous les empires de ce monde, c'est-à-dire sur l'Empire romain, ne nous ont pas été cachés : vous les venez d'apprendre de la bouche de saint Jean. Rome a senti elle-même la main de Dieu, et a été comme les autres un exemple de sa justice ${ }^{35}$. » Une fois ainsi entrevus les jugements de Dieu, l'histoire s'offre à nous comme « un grand spectacle ${ }^{36}$ » au cours duquel se révèlent les causes des progrès et de la décadence des empires ${ }^{37}$ dont l'histoire si souvent narrée a toujours un début, un milieu et une fin. C'est rien moins que cette histoire bien réelle et non pas construite par des historiens que Bossuet entend faire découvrir à son royal élève sous les traits d'une fascinante intrigue aux multiples péripéties : «Ce long enchainement des causes particulières qui font et défont les empires dépend des ordres secrets de la divine Providence. Dieu tient du plus haut des cieux les rênes de tous les royaumes. Il a tous les cœurs en sa main : tantôt il retient les passions, tantôt il leur lâche la bride, et par là il remue tout le genre humain ${ }^{38}$. "

$\mathrm{Si}$, toutefois, on avait raison d'en reconnaître l'autorité, la théologie de l'histoire rendrait donc possible la lecture cohérente d'un récit qui serait littéralement découvert dans le monde réel et qui ne serait en aucune façon imposé à celui-ci. Mais est-il nécessaire, pour découvrir ce type de récit dans l'histoire réelle, de recourir ainsi aux clés qu'un Narrateur divin nous aurait révélées dans un livre sacré. Ceux qui entendent s'en tenir à une réflexion strictement philosophique et rationnelle peuvent-ils eux aussi espérer retrouver un récit de ce genre dans l'histoire réelle? Il est

34. Augustin (saint), 1982, vol. I, p. 165.

35. Bossuet, 1901, t. III, p. 56.

36. Bossuet, 1901, t. I, p. 5 et t. III, p. 58.

37. BosSUET, 1901.

38. Bossuet, 1901, t. III, p. 156. 
certes permis d'en douter, mais ceux qui se sont adonnés à ce qu'on a appelé la « philosophie spéculative de l'histoire » ont répondu à cette question par l'affirmative ${ }^{39}$.

Il est vrai que les philosophes de l'histoire n'ont pas tous au même degré adopté une démarche explicitement narrative. Herder, dans une première œuvre rédigée en 1774, Une autre philosophie de l'histoire, est peut-être celui qui a choisi le plus volontiers de présenter l'histoire de l'humanité comme le déroulement ordonné d'une vie dont les « intrigues » sont familières à chacun de ses lecteurs. Il raconte, en effet, comment l'humanité a vécu une "enfance» orientale puis une «adolescence» marquée par l'éveil à la culture grecque avant de connaître une pleine " maturité » parfaitement incarnée dans l'Empire romain. Toutefois, ce récit trop étroitement calqué sur celui qui raconte les phases d'une vie humaine aurait fâcheusement forcé Herder à prêter au monde moderne les traits de la sénilité s'il n'avait opportunément choisi de voir dans les invasions barbares, qui ont donné le coup de grâce à l'Empire romain, l'expression d'une nouvelle jeunesse de l'humanité, qui, animée cette fois par le levain de la religion chrétienne, allait parcourir à nouveau un cycle comparable à celui de la vie humaine.

C'est toutefois Hegel qui a su le mieux traduire une vision globale de l'histoire non pas certes dans un texte explicitement narratif, mais dans un texte qui sous des dehors abstraits et hautement théoriques n'en conserve pas moins les traits essentiels d'un récit ${ }^{40}$. Ne pourrait-on pas interpréter la philosophie hégélienne de l'histoire comme étant elle-même un quasi-récit,

39. Mink, 1978, p. 188 sqq., est l'un des rares narrativistes à avoir attiré explicitement l'attention sur le fait que la « philosophie spéculative de l'histoire » - que, pour sa part, il désigne du nom de "Universal History" - suppose que l'histoire réelle est structurée comme un récit. Toutefois, dans le cadre du court article où il fait cette observation et qui est consacré avant tout à la fonction cognitive de la narration, Mink ne me semble pas avoir tiré tout le parti possible de ce constat. Voir aussi DraY, 1989, chap. VII, p. 162, où l'on trouve une brève allusion à cette question, et surtout CARROLL, 1990, p. 151-154 qui, comme on l'a vu, dénonce ce rapprochement.

40. Rıcceur, 1983, p. 205, assure pourtant que «la philosophie hégélienne de l'histoire n'est assurément pas narrative ". Cette remarque visait, toutefois, avant tout la conception assez rigide que se fait Danto de ce qu'il appelle la "philosophie substantive de l'histoire », laquelle serait caractérisée par la prétention de pouvoir énoncer des «propositions narratives » qui font appel à une connaissance du futur de l'humanité. Si tel est le propre d'une philosophie de l'histoire qui serait « narrative », Ricœur a certes raison de nier que la philosophie hégélienne de l'histoire soit narrative. Toutefois, sans prétendre connaitre le futur, la philosophie hégélienne entend bien, à la lumière de l'histoire actuelle, décrire l'essentiel du passé à l'aide de «propositions narratives », un peu comme l'historien de notre époque peut décrire la naissance d'un enfant au XVIII" siècle comme étant « en soi et pour nous » - s'il tenait à parler comme Hegel - celle du futur auteur d'une cuvre importante. Par ailleurs, quand Ricœur ajoute, dans la phrase suivante que « l'anticipation du futur dans une philosophie ou une théologie de l'espérance, n'est pas non plus narrative », il a sans doute raison, mais dans la mesure seulement où, à ses yeux, ce qu'il appelle une théologie de l'espérance a bien peu à voir avec une théologie de l'Histoire du type de celle de Bossuet. 
au sens où Ricœur n'a pas hésité à présenter comme un quasi-récit le texte beaucoup moins narratif - ne serait-ce que parce que moins soumis aux exigences d'une chronologie - de la Méditerranée de Fernand Braudel ${ }^{41}$. Pour Hegel, si la Nature n'a pas d'histoire, l'Humanité en a une et son histoire a naturellement un début, un commencement et une fin. Cette histoire s'amorcerait quelque part en Orient et, après maintes péripéties (ou « retournements » dialectiques), s'achèverait avec le triomphe de l'Esprit ou, si l'on préfère s'exprimer plus prosaïquement, avec le triomphe de la culture occidentale. Sans s'arrêter à identifier les « quasi-personnages " et les «quasi-événements " qui font de ce récit une histoire que l'on peut suivre avec intérêt, on peut mettre en relief la façon dont Hegel nous fait assister au développement d'une «quasi-intrigue». Si, selon Ricœur, c'était avant tout par le biais de «l'imputation causale singulière » que les travaux des historiens comme ceux de l'école des Annales mettaient en relief une quasi-intrigue, chez Hegel c'est plutôt par le biais des « ruses de la raison » que cette quasi-intrigue prend forme. En fait, comme le ferait tout bon historien à une échelle plus modeste, Hegel retient parmi les événements ceux qui ont une véritable portée causale en évaluant l'importance de leurs conséquences non voulues pour le développement de l'intrigue à travers laquelle l'humanité réalise son destin. Si la traversée du Rubicon peut être considérée par Hegel, tout autant que par Bossuet, comme un événement digne de mention, c'est parce qu'à travers cet événement, modeste en apparence, s'est déclenché le processus qui devait conduire à l'unification de la culture antique, laquelle devait constituer à son tour un tremplin pour le développement ultérieur de l'Occident. Dans ce contexte, les « propositions narratives » s'imposent car il serait absurde de signaler la traversée par César d'un modeste ruisseau en l'an 49 avant J.-C. si ce n'était pour présenter cet événement comme le premier pas dans la formation ultérieure de ce qui allait constituer l'Empire romain et sceller pour des siècles le sort d'une immense partie de l'humanité. De la même façon, Marx, dont la philosophie de l'histoire, sur ce plan, doit beaucoup à Hegel, présente dans le Manifeste du parti communiste, les bourgeois comme leurs «propres fossoyeurs ${ }^{42}$ » car, dans l'intrigue dont il dégage alors les grandes lignes, leur recherche fébrile de profit a pour effet de mettre en place une classe qui, dans un temps ultérieur, sera en mesure de les renverser et de les détruire. Sans doute, ces philosophies de l'histoire sont-elles devenues des philosophies «honteuses» depuis que l'Occident a pris conscience de ses limites - concernant celle de Herder et celle de Hegel - ou depuis que le communisme a échoué lamentablement - pour celle de Marx.

41. Riceur, 1983, p. 287-313. TAYLOR, 1985, p. 314, résume ce quasi-récit de Ricœur selon un mode qui pourrait fort bien s'appliquer à la philosophie hégélienne de l'histoire.

42. Karl MARX, Manifeste du parti communiste, in MARX, 1963, p. 173. 
Mais quoi qu'il en soit de leur bien-fondé et de leur contenu précis, l'élaboration de telles philosophies de l'histoire constituait une sorte d'exercice incontournable pour des penseurs d'ailleurs fort respectables qui entendaient dégager de façon correcte et définitive la signification réelle des principaux événements qui ont marqué l'histoire de l'humanité. En mettant au point de telles philosophies de l'histoire, ces penseurs estimaient pouvoir dégager ce qui s'est vraiment passé au cours de l'histoire de l'humanité. Choqués par la frivolité d'historiens qui se contentaient de découper les intrigues les plus insignifiantes du chiasme historique dont il s'agissait de rendre compte, ils entendaient bien mettre en évidence l'intrigue fondamentale qui, à leurs yeux, structurait cet ensemble historique.

On retrouve ici le problème posé par l'interprétation du cri du général de Gaulle ou de tout événement relativement récent d'importance similaire. On l'a vu, proposer une interprétation d'un événement de ce genre, c'est le situer dans le contexte historique plus large de ce qui est en train de se réaliser dans une histoire qui n'est nullement achevée. Vouloir en rendre pleinement compte, c'est donc, en dernier ressort, s'engager dans un exercice qui diffère en degré et non pas en nature de celui auquel s'adonnaient les philosophes spéculatifs de l'histoire. S'engager sur le terrain miné de l'interprétation d'événements marquants aux conséquences souvent incalculables pourrait donc équivaloir à se faire le narrateur d'une histoire dont, jusqu'à un certain point, on est forcé de supposer connue la fin encore à venir. C'est assurer que l'intrigue dans laquelle on propose d'insérer l'événement en question est une intrigue qui existe déjà dans la réalité, en ce sens du moins que son dénouement, encore à venir mais en quelque sorte inéluctable, disqualifie à l'avance toutes les intrigues concurrentes qui auraient pu en réduire la portée. Inversement, dire que le monde de l'action humaine a une structure narrative que les historiens devraient dégager sans chercher à lui imposer une intrigue de leur cru, équivaut à cautionner le bien-fondé d'une philosophie de l'histoire, car une philosophie de l'histoire n'est rien d'autre que la mise en lumière de l'intrigue fondamentale dont la connaissance du dénouement permettrait de fixer le sens de développements historiques qui, autrement, pourraient toujours être interprétés et narrés de multiples façons. Adopter une philosophie de l'histoire, comme le faisaient tant de marxistes hier encore, ou comme le fait volontiers aujourd'hui un penseur comme Francis Fukuyama ${ }^{43}$, c'est refuser aux historiens la liberté d' «imposer » à la réalité historique des intrigues que ne cautionneraient pas le sens de l'histoire mis en lumière par cette philosophie.

43. Fukuyama, 1992. 
Il est clair que Carr, qui n'a pas manqué de prendre clairement ses distances à l'égard de la philosophie spéculative de l'histoire ${ }^{44}$, n'accepterait pas cette réinterprétation du réalisme qu'il prône à propos du récit historique. Aussi, serait-il bien injuste d'associer son nom à ce type de spéculation sur l'histoire. Toutefois, il me semble que l'on retrouve dans les travaux des philosophes spéculatifs de l'histoire une expression différente de l'élément le plus incontournable de sa thèse et peut-être la seule façon de la rendre pleinement cohérente sinon vraiment défendable. En effet, Carr a raison de soutenir que l'histoire ne saurait être une masse informe à laquelle l'historien « imposerait » de l'extérieur les intrigues qu'il lui plaît d'y voir se développer. Il doit bien y avoir dans l'histoire réelle des intrigues plus significatives que d'autres, des intrigues mieux ancrées que d'autres dans ce qui s'est effectivement passé au cours de l'histoire, ou mieux dans ce que l'histoire a effectivement permis de réaliser. Mais admettre cela n'implique nullement que ces intrigues préexistaient en quelque sorte dans les plans que les principaux acteurs de l'histoire avaient en vue. On peut soutenir qu'il s'est effectivement passé quelque chose de significatif au cours de l'histoire, que quelque chose s'y est manifestement réalisé - c'est ce que font en particulier les politologues et les praticiens des sciences sociales qui analysent les «conséquences non voulues des phénomènes »-, sans prétendre que c'est ce que les acteurs de l'histoire ont voulu réaliser ou même ce qu'ils auraient immédiatement inscrit, de façon non équivoque, dans l'histoire. Toutefois, se prononcer ainsi sur ce qui est en train de se réaliser dans une histoire qui en fait n'est pas encore achevée, c'est tenter de faire pour un segment de l'histoire un peu arbitrairement détaché du contexte de l'histoire universelle, ce que cherchaient à faire, à une échelle ostensiblement et prétentieusement universelle, les philosophes spéculatifs de l'histoire.

Même réduite aux dimensions de celle du politologue qui veut dégager la portée du «Vive le Québec libre ! » du Général, une telle ambition ne se heurte pas moins à la difficulté que Danto opposait à toute philosophie « substantive » (ou spéculative) de l'histoire ${ }^{45}$, dans la mesure, du moins, où celle-ci prétend juger du présent à la lumière d'un avenir encore inconnu, en décrivant ce présent à l'aide de «propositions narratives » qui font appel à un état de chose futur ou, si l'on préfère, à une « fin de l'histoire ». Peut-être est-ce dans ce contexte qu'il faut comprendre le désarroi manifesté par Maurice Merleau-Ponty au moment où il venait de s'éloigner résolument du marxisme et, du même souffle, de rejeter définitivemenı l'idée même qu'il soit possible d'invoquer une quelconque philosophie de

44. CARR, 1986, p. 1; voir aussi sa discussion de Hegel au chap. vı de CARR, 1986, p. 159 160.

45. Danto, 1968 , chap. I. 
l'histoire, lui qui, peu de temps auparavant, avait vu dans la philosophie marxiste de l'histoire la seule voie susceptible de permettre une compréhension réelle des événements historiques ${ }^{46}$. Il faut reconnaître, en tout cas, qu'il est manifestement beaucoup plus difficile qu'on ne le croit généralement de nos jours de se passer, avec cohérence, de philosophie spéculative de l'histoire même s'il est de plus en plus difficile d'en adopter une de façon crédible.

Faut-il en conclure, vu le scepticisme qu'inspire généralement et légitimement toute philosophie spéculative de l'histoire qui ose s'afficher comme telle, que la notion même d'histoire se trouve en péril, si l'on peut s'exprimer ainsi à son propos? Pas vraiment, si l'on veut seulement admettre que, dans la mesure où elle entend rendre compte du déploiement dans le temps des activités humaines, l'histoire serait menacée bien davantage par une philosophie de l'histoire qui certes lui apporterait une structure permettant de la saisir comme totalité, mais non sans détruire cela même qui la définit, soit cette ouverture sur un avenir qui diffère radicalement du présent en ceci qu'il n'est pas donné et ne peut être connu. C'est ainsi que ce trait essentiel de la temporalité ou, si l'on préfère de l'historicité, dont Carr rappelait fort éloquemment l'importance, n'est guère compatible avec le genre de philosophie de l'histoire qui, en dernier ressort, semble requis pour qu'il y ait un sens acceptable à soutenir, comme Carr, que le monde historique en tant que tel est doté d'une structure narrative analogue à celle qui sous-tend les histoires narrées par les historiens.

Peut-être nous heurtons-nous là à une sorte de paradoxe constitutif de l'histoire, laquelle vise forcément à exprimer de manière exacte — et en ce sens définitive - l'état d'inachèvement d'un monde inachevé, du fait qu'il demeure toujours susceptible de se révéler autre que ce que, en cet état d'inachèvement, il laisse penser qu'il sera. Certes, la tentation est grande alors d'adopter un point de vue qui a la prétention de saisir ce monde dans son achèvement et de décrire avec Hegel la façon dont se présente ce monde de ce point de vue, c'est-à-dire « en soi ou pour nous », mais la philosophie de l'histoire qui est ainsi évoquée n'est autre qu'une structure, Hegel l'a bien vu, qui est fort apparentée à celle d'une Phénoménologie de l'esprit, voire à celle d'une Logique. Or ce qui est ainsi gagné sur le plan structurel, on l'a bien vu depuis Hegel, risque fort de n'être acquis qu'au prix du temps et de la contingence, bref, de n'être acquis qu'au prix de l'histoire elle-même. Poussé à la limite, l'acte de configuration - qui caractérise une narration ne pouvant se développer qu'au sein de la temporalité - se transformerait alors en cette synthèse intemporelle que Mink, à

46. Voir à ce sujet LaGueux, 1966. 
juste titre, associait au totum simul de Boèce ${ }^{47}$, c'est-à-dire à la négation, dans l'absolue contemporanéité d'une perception divine, de toute temporalité et de toute historicité. Pour échapper à cette totalisation négatrice de la temporalité, il semble bien qu'il y ait un prix à payer : celui de la contingence radicale d'une histoire qui, ontologiquement, ne serait pas plus structurée que le chiasme de relations inépuisables dont il a été question ici. Mais, inversement, pour échapper à l'éparpillement insignifiant d'une histoire sujette aux lectures les plus arbitraires, il semble bien qu'il y ait aussi un prix à payer : il consiste à entrouvrir discrètement mais dangereusement la porte aux philosophies spéculatives de l'histoire.

Maurice LagueuX (septembre 1996).

47. Mink, 1970, p. 549. 
BIBLIOGRAPHIE

ArIstote, 1990, Poétique, trad. Michel MaGnIEN, Paris, Librairie générale française (Le Livre de poche, 6734. Classique).

Augustin (saint), 1994, La Cité de Dieu, trad. du latin de Louis Moreau (1846), rev. par Jean-Claude Esun, Paris, Seuil.

Bossuet (Jacques Bénigne), 1901, Discours sur l'histoire universelle, éd. L. PfluGER, Paris, Librairie de la Bibliothèque nationale.

Canary (Robert H.), Kozicki (Henry), 1978, The Writing of history. Literary form and historical understanding, Madison, University of Wisconsin Press.

CArr (David), 1985, “Table ronde/Round Table », Revue de l'université d'Ottawa, vol. 55, 4, p. 301-311.

CARR (D.), 1986, Time, narrative and history, Bloomington, IN, Indiana University Press.

Carr (D.), 1991, «Épistémologie et ontologie du récit », in Greisch, Kearney, 1991, p. 205-214.

Carroll (Noël), 1990, «Interpretation, history and narrative », The Monist, vol. 73.

Danto (Arthur), 1968, $1^{\text {re }}$ éd. 1965, Analytical Philosophy of history, New York, Cambridge University Press.

DraY (William), 1989, On history and philosophers of history, Leyde/New York, E. J. Brill.

FukuYama (Francis), 1992, The End of history and the last man, New York, Macmillan, The Free Press.

Gallie (Walter B.), 1964, Philosophy and the historical understanding, Londres, Chatto \& Windus.

Greisch (Jean), Kearney (Richard), 1991, Paul Ricaur. Les métamorphoses de la raison herméneutique, Paris, Cerf.

Hegel (Georg Wilhelm Friedrich), 1939, La Phénoménologie de l'esprit, trad. Jean HYPPOLITE, Paris, Aubier-Montaigne.

LagueuX (Maurice), 1966, «Y a-t-il une philosophie de l'histoire chez MerleauPonty? », Dialogue, V, p. 404-417.

MarX (Karl), 1963, Euvres. T. I : Économie, Paris, Gallimard (Bibliothèque de la Pléiade, 164).

Mink (Louis), 1970, « History and fiction as modes of comprehension », New Literary History. A journal of theory and interpretation, printemps 1970, p. 541558.

Mink (L.), 1978, «Narrative form as a cognitive instrument », in Mink, 1987, p. 182-203; paru d'abord in Canary, KozICKI, 1978, p. 129-149.

Mink (L.), 1987, Historical Understanding, Ithaca, Cornell University Press.

Ricceur (Paul), 1983, Temps et récit, t. I, Paris, Seuil.

RiCceur (P.), 1985, «Table ronde/Round table », Revue de l'université d'Ottawa, vol. 55,4 , p. $316-322$. 
TAYLOR (Charles), 1985, «Table ronde/Round table», Revue de l'université d'Ottawa, vol. 55,4 , p. 311-316.

WhITE (Hayden), 1973, Metahistory. The historical imagination in nineteenthcentury Europe, Baltimore, MD, Johns Hopkins University Press.

White (H.), 1978, "The historical text as literary artifact », in CANARY, KozIcKI, 1978, p. 41-62. 